\title{
REVISTAMATĒRIA
}

ISSN 1517-7076 artículo e-12020, 2018

\section{Espuma ultraliviana de Mg a partir de viruta reciclada}

\author{
Ultra lightweight Mg foam from \\ recycled shavings
}

\author{
Carlos Oldani ${ }^{1}$, Luciano Grinschpun ${ }^{1}$, Matías Schneiter ${ }^{1}$, \\ Rodrigo Milne ${ }^{1}$,Daniel Acciarri ${ }^{1}$, Noam Abadi ${ }^{1}$
}

\author{
${ }^{1}$ Departamento de Materiales y Tecnología, Facultad de Ciencias Exactas, Físicas, y Naturales, Universidad Nacional de \\ Córdoba, Av. Vélez Sarsfield 1611, Córdoba, Córdoba, Argentina. \\ e-mail: carlos.oldani@unc.edu.ar
}

\section{RESUMEN}

Cientos de personas en el mundo mueren o quedan seriamente dañadas a causa de colisiones en accidentes de tránsito, debido a la energía que se transmite a los ocupantes del auto por el impacto. Para minimizar esta energía, es necesario disiparla mediante absorbedores. En años recientes, se empezaron a considerar las espumas metálicas como absorbedores de energía. Las espumas metálicas son materiales estructurales relativamente desconocidos, pero con un enorme potencial para aplicaciones donde el bajo peso combinado con alta rigidez son de principal interés. El uso de espumas ultra-livianas de magnesio ( $\mathrm{Mg}$ ) o sus aleaciones, permitirían cumplir con dos requisitos: disminución de peso (mayor eficiencia y menor polución) y absorción de energía de impacto (menores daños a los pasajeros del vehículo).

El mecanizado de cajas de cambio de aleación de magnesio AZ91D en la indústria autopartista, genera una gran cantidad de viruta contaminada con aceites. En este trabajo se usa la metalurgia de polvos como método para la recuperación de la viruta de aleación AZ91D. Se estudiaron las condiciones pulvimetalúrgicas para la obtención de una espuma a partir de viruta y separador (bicarbonato de amonio). El material obtenido se caracterizó físicamente determinando la densidad de la espuma y mecánicamente mediante ensayos de compresión, obteniendo valores de energía absorbida y energía absorbida específica, que son los datos que caracterizan una espuma. Finalmente, se lo caracterizó metalográficamente utilizando microscopía óptica y electrónica confocal. Se probaron distintos porcentajes de porosidad (50 y 70\%). La energía absorbida por unidad de masa $(\mathrm{J} / \mathrm{g})$ de la espuma con $50 \%$ de porosidad resultó ser mayor al doble de la absorbida por el magnesio sinterizado sin separador

Palabras clave: Espumas metálicas, absorbedores, energía.

\section{ABSTRACT}

Hundreds of people worldwide die or are severely maimed in collisions during traffic accidents, due to the energy transmitted to the passengers on impact. A dissipation of said energy is necessary to minimize it's effects, which can be achieved through absorbers. In recent years, metallic foams have started to be considered as energy absorbers. Metallic foams are relatively unknown structural materials, but with huge potential for applications where low weight but high rigidity are of primary interest. The use of ultralight magnesium $(\mathrm{Mg})$ or magnesium alloy foams has two main advantages: less weight (more vehicle efficiency and less pollution) and impact energy absorption (less harm to passengers on board the vehicle).

The machining of gearboxes made out of AZ91D magnesium alloy, generates large amounts of oilcontaminated metallic shavings. In this work, powder metallurgy is used as a recycling method of the AZ91 alloy. There were studied the conditions needed for obtaining metallic foam from recycled shavings of AZ91 and spacer (ammonium bicarbonate). The final product was characterized physically by determination of the foam's density. Also, compression tests were done to obtain values of absorbed energy, and specific absorbed energy, which characterize a metallic foam. Different metallography methods were used to characterize the structure of the sintered material. Different porosities were tested (50 and 70\%). The foam's absorbed energy by unit mass $[\mathrm{J} / \mathrm{g}]$ of the $50 \%$ of porosity foam was over double compared to that of the magnesium sintered 
without spacer.

Keywords: Metallic foams, energy absorbers.

\section{INTRODUCCIÓN}

De todos los metales estructurales, el magnesio es el de menor densidad $\left(1,73 \mathrm{~g} / \mathrm{cm}^{3}\right)$, razón por la cual sus aleaciones se utilizan cada vez más en autopartes. El mecanizado de cajas de cambio de aleación de magnesio AZ91D genera una gran cantidad de viruta contaminada con aceites.

Se conocen diversos métodos para reciclar viruta de magnesio, siendo la fusión el más importante. Sin embargo, el proceso es complicado ya que debe hacerse con protección del baño líquido mediante gases (generalmente tóxicos) o mezcla de sales $[1,2,3]$. En este trabajo se usa la metalurgia de polvos como método alternativo para la recuperación de la viruta de aleación AZ91 [4,5]. Este proceso comprende la preparación y mezcla de polvos de la aleación metálica y su posterior compactación para obtener un "verde" que pueda manipularse con facilidad. En la mezcla del polvo de AZ91 se agregan partículas de un agente formador de poros (separador), como el bicarbonato de amonio, que sublima a temperaturas relativamente bajas dejando espacios libres en la estructura y generando un material poroso (espuma).

Las espumas metálicas son materiales relativamente desconocidos, aunque poseen una combinación única de propiedades, tales como alta capacidad de absorción de energía de impacto durante el colapso de los poros [6], buenas propiedades de amortiguación, permeabilidad de aire y agua, propiedades acústicas, baja conductividad térmica y buen aislamiento eléctrico [7]. En la indústria automotriz, el uso de espumas ultralivianas de magnesio ( $\mathrm{Mg}$ ) o sus aleaciones, con una densidad entre $0,5-1,5 \mathrm{~g} / \mathrm{cm}^{3}$, permitirían cumplir con dos requisitos: disminución de peso (mayor eficiencia y menor polución) y absorción de energía de impacto (menores daños a los pasajeros del vehículo) $[8,9,10]$.

El objetivo de este trabajo es encontrar las condiciones pulvimetalúrgicas adecuadas para la obtención de una espuma a partir de viruta reciclada de aleación AZ91 y su caracterización.

\section{MATERIALES Y MÉTODOS}

Para la realización del trabajo se utilizó viruta de aleación de magnesio AZ91D proveniente del mecanizado de esta aleación, limpiada según el procedimiento desarrollado por Milne [5]. Como separador y generador de poros se usó bicarbonato de amonio marca Anedra, de $99.6 \%$ de pureza y con tamaños de partículas menores a $90 \mu \mathrm{m}$. La viruta limpia debe ser molida y compactada para realizar el sinterizado del proceso pulvimetalúrgico.

El primer paso en el proceso es la molienda de la viruta, la que presenta forma de laminillas cuyo tamaño original varía entre los $2.5 \mathrm{~mm}$ y $10 \mathrm{~mm}$ de diámetro medio aproximadamente. Esto se realizó en un molino planetário a bolas durante 15 minutos. Para evitar la oxidación de las partículas de polvo, se realizó un barrido con gas argón de las vasijas del molino, evacuando el oxígeno. La granulometria del polvo metálico obtenido se encuentra principalmente en un tamaño de partícula menor a los $62 \mu m$.

Una vez obtenido el polvo de la viruta, se lo mezcló con el separador de modo de fijar un determinado porcentaje en volumen de poros. Se trabajó con porosidades de 50 y $70 \%$. Para la mezcla de los polvos se utilizó un mezclador en Y, rotando durante 15 minutos.

Posteriormente la mezcla se comprimió en una matriz cilíndrica de $8 \mathrm{~mm}$ de diámetro interno, con una presión de $585 \mathrm{MPa}$, de donde se obtiene una muestra ("verde") relativamente rígida que permite manipulación leve.

Para el sinterizado se utilizó una cámara diseñada para minimizar el espacio libre y asegurar una buena atmósfera protectora de argón (Figura 1). de argón:

El proceso de sinterizado usado consistió en las siguientes etapas, todas realizadas bajo una atmósfera

- $\quad$ Calentamiento (con una velocidad de aproximadamente $10^{\circ} \mathrm{C} / \mathrm{min}$ ) desde temperatura ambiente hasta una temperatura de $200^{\circ} \mathrm{C}$.

- Mantenimiento a temperatura constante durante 30 minutos para permitir la sublimación del separador.

- $\quad$ Calentamiento hasta la temperatura de sinterizado $\left(600^{\circ} \mathrm{C}\right)$ y permanencia a esta temperatura durante 2 horas. 


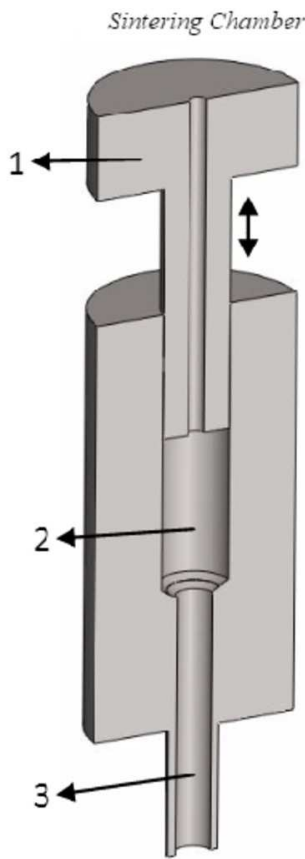

Figura 1: Cámara de sinterizado: 1. Tapa, 2. Cavidad donde se aloja la muestra a sinterizar, 3. Entrada de argón, 4. Orificio para salida de argón.

\section{RESULTADOS Y DISCUSIÓN}

\subsection{Metalografía}

Las muestras porosas sinterizadas fueron analizadas metalográficamente para determinar, a partir de microfotografías y mediante cálculo, cuál era la porosidad resultante final. En la Figura 2 se presenta, a modo de ejemplo, la micrografía de una muestra realizada bajo el procedimiento descrito, para el $50 \%$ en volumen de separador (bicarbonato de amonio). Para calcular la porosidad obtenida luego del sinterizado se utilizaron tintas penetrantes antes del pulido final, con el fin de aumentar el contraste entre poros y matriz y de esta manera mejorar el cálculo de la porosidad. Los resultados indican una porosidad media de $56,4 \pm 4,4 \%$ para la muestra de $50 \%$. La variación de la porosidad en la dirección longitudinal varía entre 40-70\%, aunque no se ve una tendencia que dependa de la dirección. El método óptico (metalográfico) de medir la porosidad representa bien los valores experimentales. Se puede ver que las cantidades agregadas de separador se corresponden bien con los valores de porosidad buscados.

La metalografía muestra la presencia de poros aislados e interconectados, con formas irregulares, pero con una tendencia a ser alargados en la dirección radial (horizontal). La alta variación de porosidad puede ser producto de la alta hidroscopicidad del bicarbonato de amonio, la cual promueve la aglomeración de partículas. Por otro lado, la sublimación gaseosa del separador favorece la predominancia de poros abiertos e interconectados. La misma variación en el porcentaje de poros y en las características morfológicas se repiten en las muestras con $70 \%$ de porosidad.

Los poros tienen una geometría compleja que presenta interconecciones. Estas descontinuidades producen concentración de tensiones en las paredes internas de los poros lo que hace que, cuanto mayor sea el porcentaje de porosidad, más cerca van a estar los poros entre si produciéndose un incremento aún mayor en esta concentración de tensiones [13].

En las muestras con porosidad por encima del $70 \%$, el material queda totalmente frágil y se desgrana con la mano luego del sinterizado. Esto es posible que se deba a dos causas: por una parte, el bicarbonato de amonio sublima por encima de $\operatorname{los} 35^{\circ} \mathrm{C}$, descomponiéndose en amoniaco, dióxido de carbono y agua. La presencia de agua podría aportar el oxígeno necesario para la oxidación de las partículas de polvo de magnesio no permitiendo el sinterizado. A esto se le suma el efecto de los fuertes concentradores de tensión. 
Para describir las propriedades mecánicas del material poroso se comprimió cada muestra (ensayo de compresión cuasi-estático), se determinó la capacidad de absorción de energía según el modelo utilizado por YU, S. et al [11] y se calculó la absorción de energia por unidad de masa.

Idealmente se espera que un material poroso tenga un menor módulo de elasticidad respecto al metal de la matriz debido a la presencia de los poros (que pueden considerarse como partículas con módulo de elasticidad nulo). De la misma manera, la tensión de fluencia disminuirá en el material poroso.

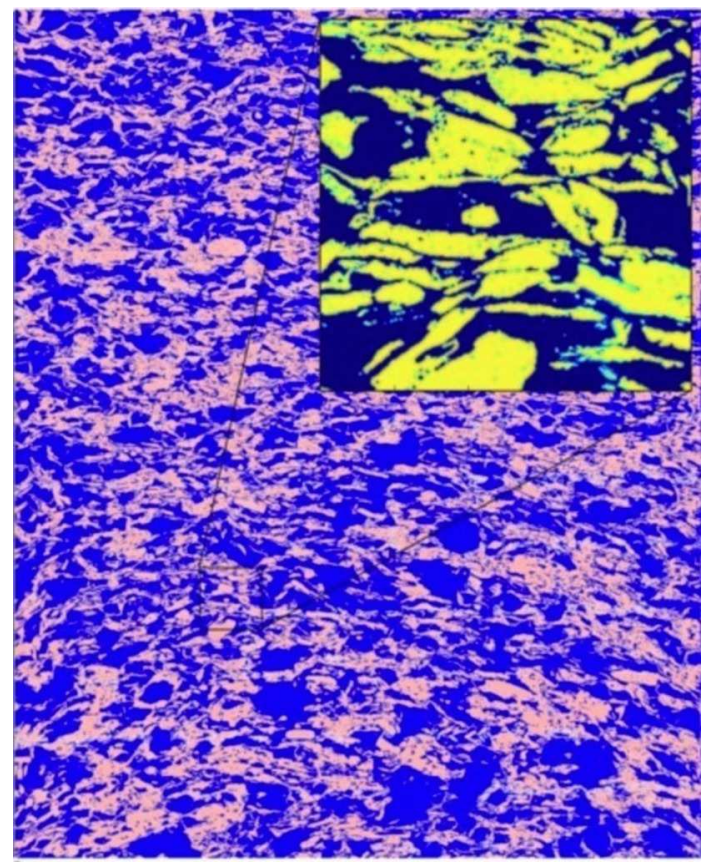

Figura 2: Micrografía de la muestra sinterizada con $50 \%$ de porosidad, con un detalle de 6 veces el tamaño original.

En la figura 3 se comparan las curvas de tensión-deformación para las muestras de 50\% y $70 \%$ de porosidad. Como puede observarse, las curvas muestran un comportamento similar al de un material poroso ideal, con una región de plateau característico [12].

La Capacidad de absorción de energia se calculó utilizando la ecuación 1[8], hasta una deformación específica, $\varepsilon$ del 0.7 :

$$
W=\int_{0}^{\epsilon} \sigma d \epsilon
$$

donde $\boldsymbol{W}$ es la capacidad de absorción de energía.

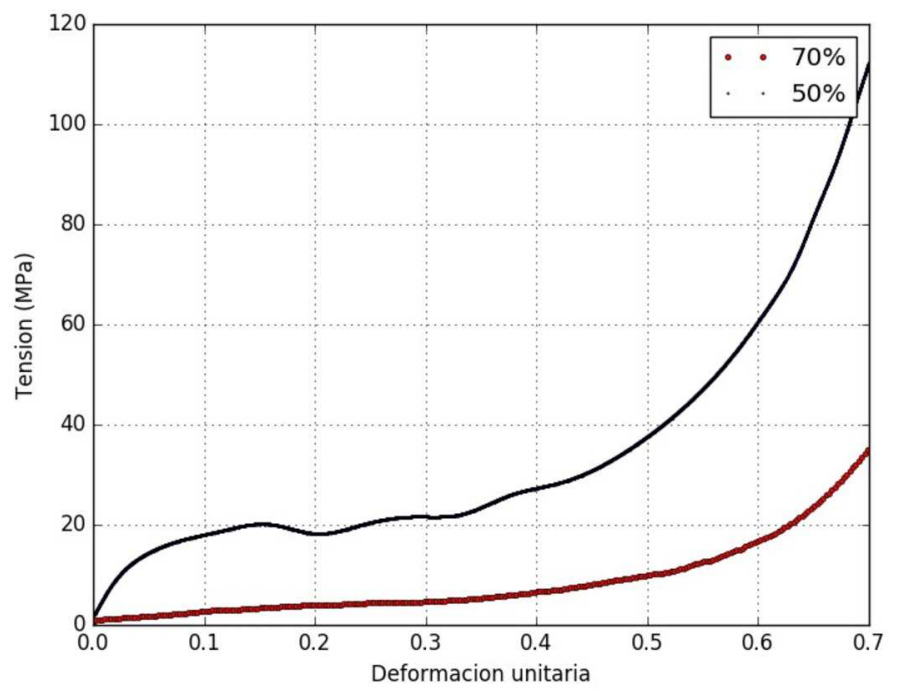


Figura 3: Curvas tensión-deformación para las muestras de 50\% y 70\% de porosidad.

La figura 4 muestra la capacidad de absorción de energía para espumas de 50 y $70 \%$ de porosidad. Los valores de energia absorbida encontrados son similares a los que presentan las espumas comerciales de alumínio utilizadas como absorbedores [13], para el caso del $50 \%$ de porosidad (densidade relativa $=0.5$ ). Sin embargo, al ser menor la densidad del magnésio, éste presentaría uma ventaja técnica competitiva.

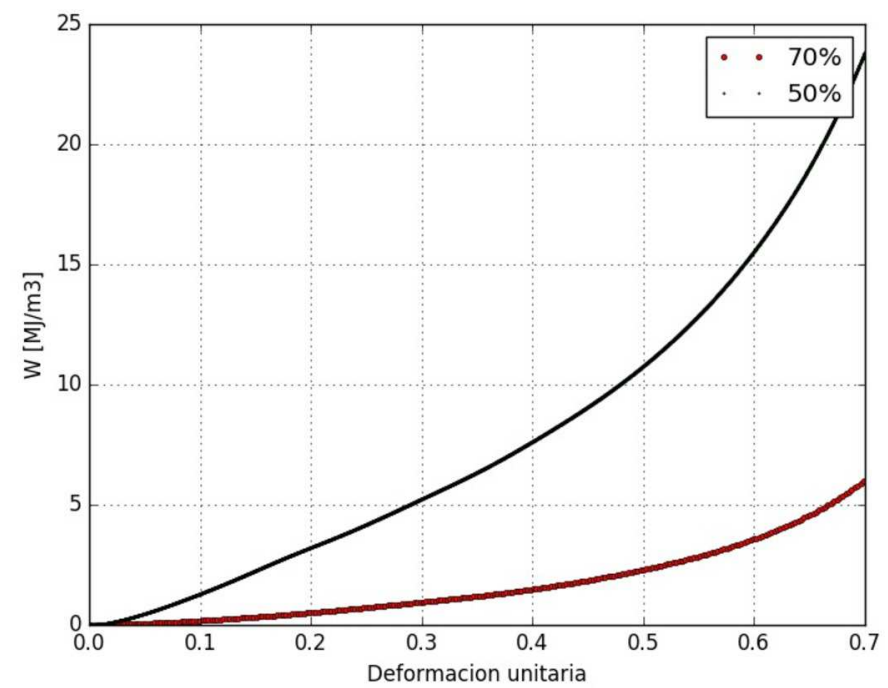

Figura 4: Capacidad de absorción de energía en función de la deformación para muestras con 50\% y 70\% de porosidad.

En la Tabla 1 se representan los valores obtenidos de energía absorbida específica $(\mathrm{W} / \delta)$ y energia absorbida por unidad de masa $(\mathrm{W} / \mathrm{m})$, para las dos espumas con diferentes porcentajes de porosidad, comparados con los valores del material sinterizado sin poros (sin agregado de separador).

Como se ve de la Tabla 1, un material con porosidad de $0 \%$ (material no poroso) presenta una absorción de energía baja, mientras que un material con un porcentaje de porosidad muy elevado implica una absorción de energía casi nula debido a la escasa masa del metal de la matriz. Esto mostraría que debe existir un valor máximo de absorción de energia para algún valor de porosidad, disminuyendo al variar ésta.

Es interesante destacar que una misma masa de AZ91 en forma de espuma con $50 \%$ de porosidad absorbe el doble de energia que una muestra de material homogeneo sin porosidad.

Tabla 1: Propiedades físicas y mecánicas de espumas de AZ91D para distintas porosidades. 


\begin{tabular}{|c|c|c|c|c|}
\hline$\% \rho$ & $\begin{array}{c}\boldsymbol{\delta} \\
{\left[\mathbf{g} / \mathbf{c m}^{3}\right]}\end{array}$ & $\mathbf{m}[\mathbf{g}]$ & $\begin{array}{c}\mathbf{W} / \boldsymbol{\delta} \\
{\left[\mathbf{J} . \mathbf{c m}^{3} / \mathbf{g}\right]}\end{array}$ & $\begin{array}{c}\mathbf{W} / \mathbf{m} \\
{[\mathbf{J} / \mathbf{g}]}\end{array}$ \\
\hline 0 & 1,6 & 0,8 & 8,6 & 17,2 \\
\hline 50 & 0,8 & 0,4 & 16,1 & $\mathbf{3 4 , 5}$ \\
\hline 70 & 0,48 & 0,24 & 5,52 & 11,04 \\
\hline
\end{tabular}

\section{CONCLUSIONES}

Del trabajo realizado se puede concluir que:

Es posible utilizar el processo pulvimetalúrgico para obtener un material con alto grado de porosidad. El tamaño de los poros y su cantidad está intimamente relacionado con la cantidad y tamaño de las partículas espaciadoras agregadas de bicarbonato de amonio. Los poros generados son irregulares, alargados en la dirección radial, mayormente interconectados, y en una cantidad levemente mayor a la planeada.

Las muestras obtenidas usando la aleación AZ91D como matriz metálica y bicarbonato de amonio como separador, en porosidades de hasta $70 \%$ tienen un comportamiento lo suficientemente parecido al de una espuma ideal como para ser consideradas tales.

La densidad de las espumas estudiadas están en valores muy bajos, entre 0,48 a $0,8 \mathrm{~g} / \mathrm{cm}^{3}$.

Los valores de energia absorbida específica $(\mathrm{W} / \delta)$ son similares a los que presentan las espumas comerciales de alumínio.

\section{BIBLIOGRAFÍA}

[1] LUCCI, R., PADILLA, R. L., NICOLA, P., et al., Possibilities of Recovering AZ91 Magnesium Alloy from Machining Shavings Contaminated with Oil, through their Fusion with Argon Gas Protection, Procedia Materials (2011): 11th International Congress on Metallurgy \& Materials SAM/CONAMET 2011, disponible en www.sciencedirect.com.

[2] LUCCI, R., PADILLA, R. L., CANTERO, S., et al., Refining of AZ91 Magnesium Alloy Obtained in Machining Chips Recycling, Procedia Materials Science (2014): International Congress of Science and Technology of Metallurgy and Materials, SAM - CONAMET 2013, disponible en www.sciencedirect.com.

[3] LUCCI, R., Reciclado de magnesio: recuperación por fusión de la aleación AZ91 a partir de virutas de mecanizado y evaluación de sus propiedades, Tesis de Doctor en Ciencias, FCEFyN/UNC, Córdoba, Argentina, 2015.

[4] MILNE, R., GRINSCHPUN L., OLDANI, C., et al., Sinterizado de viruta de magnesio AZ91D, Anales del IV Congreso Argentino de Ingeniería Mecánica (CAIM), pp. 182, 2014.

[5] MILNE, R., "Recuperación pulvimetalúrgica de aleación de magnesio AZ91D con fines estructurales", Revista Facultad de Ciencias Exactas, Físicas y Naturales, v. 3, n. 2, pp. 37-43 Sep 2016.

[6] TARLOCHAN, F., SAMER, F., "Design of thin wall structures for energy absorption applications: design for crash injuries mitigation using magnesium alloy", International Journal of Research in Engineering and Technology, v. 2, n. 7, pp. 24-36, Jul. 2013.

[7] WEN, C., YAMADA, Y., SHIMOJIMA, K., et al., "Effects of heat treatment on the compressive properties of AZ91 Mg foam”, Materials Transactions, JIM, v. 41, n. 9, pp.1192-1195, Aug. 2000.

[8] SIMANČÍK, F., "Metallic foams-ultra light materials for structural applications", Inźynieria Materiałowa, v. 5, pp.823-828., May 2001.

[9] GUPTA, N., LUONG, D. D., CHO, K. "Magnesium matrix composite foams-density, mechanical properties, and applications", Metals, v. 2, n. 3, pp. 238-252, Jul. 2012.

[10] ZHAO, Y.Y., TAO, X.F., "Behaviour of metal matrix syntactic foams in compression", Material science and technology, pp. 1785-1793, Oct. 2009.

[11] YU, S., LIU, J., WEI, M., et al., "Compressive property and energy absorption characteristic of opencell ZA22 foams", Materials \& Design, v. 30 n. 1, pp.87-90, Jan. 2009. 
[12] GIBSON, LJ: Mechanical behavior of metallic foams, Annual review of materials science v. 30, n. 1 pp. 191-227, ene 2000

[13] SHEN, H., BRINSON, L.C., "Finite element modeling of porous titanium”, International Journal of Solids and Structures, v. 44, n. 1, pp. 320-335, Jan. 2007 